\title{
Soft Demapping and Turbo Decoding for Satellite Broadcasting Communications
}

\author{
Rosalba Suffritti, Enrico Del Re, Simone Morosi \\ CNIT-University of Florence \\ Department of Electronics and Telecommunications \\ Via di S. Marta 3, Firenze 50139 Italy \\ E-mail: $\{$ rosalba.suffritti, enrico.delre, simone.morosi $\} @$ cnit.it \\ phone/fax: +39055 4796485
}

\begin{abstract}
In this paper an original detection strategy for satellite digital broadcasting communications is defined; particularly, we consider a system derived from the DVB-S2 standard, which has been proposed as a development of the DVB-S system and exploits an iterative decoding and higher order modulations. The proposed approach relies on the use of the information which can be obtained by proper soft demapping schemes and on the use of the Turbo codes instead of the LDPC (Low Density Parity Check) codes required by the standard. Moreover, an original receiver which is based on iterative demapping and decoding, is introduced. The adoption of this strategy can permit a remarkable performance gain and an improvement of the system throughput.
\end{abstract}

\section{INTRODUCTION}

In recent years, the satellites have assumed a key role in order to link users at large distance or in cases where the cable connection is unpractical or uneconomic. Their use is become essential in case of mobile users spread over a large area, in particular for aeronautical and maritime communications. Moreover, they allow the extension of the range of terrestrial fixed and mobile networks, which can be primary in case of emergency situations, as well. The DVB-S2 (Digital Video Broadcasting - Satellite - Second Generation) standard [1] [2], proposed by DVB project, is a system aimed at providing a variety of satellite applications, higher power and bandwidth efficiency. The DVB-S2 system was introduced to improve the performance which is obtained by DVB-S systems; its main characteristics are the following: a flexible input stream adapter, suitable for operation with single and multiple formats of the input streams, a powerful FEC system based on LDPC (Low-Density Parity Check) codes concatenated with BCH codes, a wide range of code rates and 4 constellations which are optimized for non-linear transponders.

The introduction of higher order modulation schemes in the DVB-S2 standard permits to analyze the benefits which can be obtained using the soft and iterative detection strategy in this scenario; to this end, we have generalized the approach which has been described in [3]: this is the main original contribution of this paper. Moreover, in order to allow the application of the Turbo principle to this system, we have introduced and tested a well-known 8 -state Turbo Code system, which has been previously considered in the DVB-RCS standard [4]. The proposed system can permit to achieve remarkable results with a moderate complexity increase.

This paper is organized as follows. In Section II the system model is presented and the Turbo principle is described. Moreover the "tail-biting" technique and the "Duo-Binary Turbo Codes" theory are introduced and analyzed. In Section III the structure of the proposed receiver is shown while in Section IV the simulation results are presented. The concluding remarks are given in Section V.

\section{SySTEM MODEL}

The successful proposal of Turbo codes [5] suggests the idea of an iterative Turbo processing in the design of satellite systems, as it was adopted in DVB-RCS standard [4]. The considered Turbo Codes are composed of two Recursive Systematic Convolutional (RSC) codes connected by an interleaver. Moreover, in order to consider the input stream format flexibility and the wide range of code rates of the DVB-S2 system, we propose two structures: while the former (Fig. 1) is used for every code rate required by the standard, the latter (Fig. 2) is introduced only when the code rate is higher than $1 / 2$. The two structures are different for the number of systematic streams which are transmitted: while in the former case the data stream is only one, in the latter one the systematic streams are two, that is the data input is a couple of bits. Two different Parallel Concatenated Convolutional Code (PCCC) encoders are devised: one is a classical convolutional binary code while the other is called "duo-binary" convolutional code [6]. The "Duo-binary Turbo codes" theory [7], [8], is a generalization of the classical Binary Turbo Codes theory. In practice, for the construction of a duo-binary turbo code, a parallel concatenation of two identical RSC encoders with 2 bits word interleaving, is considered. As for the interleaver, in this case, the permutation function is performed on two levels:

- an inter-symbol permutation that modifies the order of the symbols. This rule is almost regular. It is based on faint vectorial fluctuations around the locations given by regular permutation.

- an intra-symbol permutation that reverses the bits in some prearranged couples. 


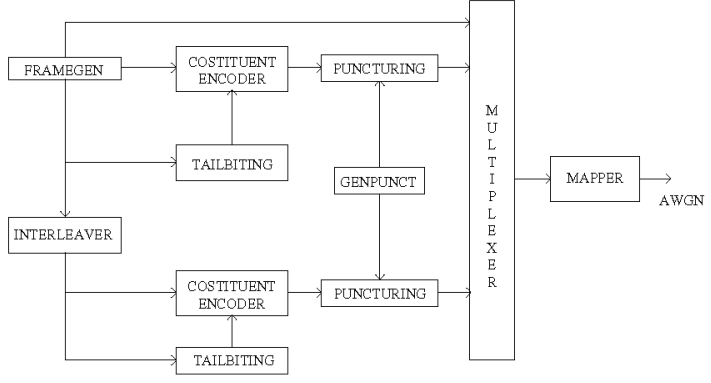

Fig. 1. Transmitter for the proposed system: binary structure

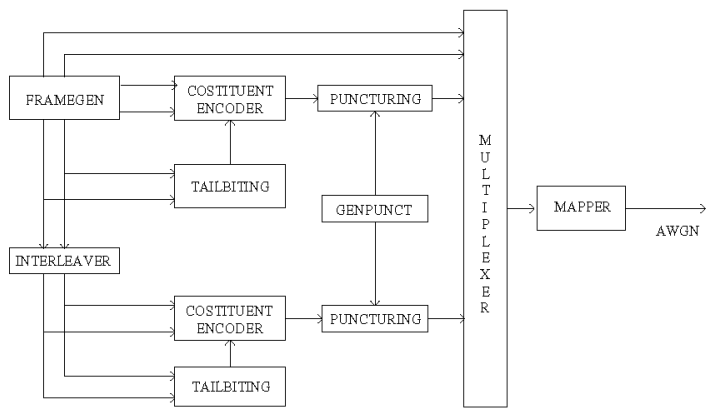

Fig. 2. Transmitter for the proposed system: duobinary structure

In order to encode data blocks without any rate loss or performance degradation due to direct truncating, in both structures the tail-biting termination which allows to view the code trellis as a circle, is adopted. This technique transforms a convolutional code into a block code allowing any state of the encoder as the initial state and enconding the sequence so that the final state of the encoder is equal to the initial state [9], [10]. There is no need to force the encoder back to the all zero-state by appending a block of tail bits to the information vector (zero termination). If we consider a rate$k_{0} / n_{0}, k_{0}<n_{0}$, convolutional encoder as a device which generates the $n_{0}$-tuple

$$
\mathbf{v}_{t}=\left(v_{t}^{(1)}, v_{t}^{(2)}, \ldots, v_{t}^{\left(n_{0}\right)}\right)
$$

of coded bits at time $t$ given the $k_{0}$-tuple

$$
\mathbf{u}_{t}=\left(u_{t}^{(1)}, u_{t}^{(2)}, \ldots, u_{t}^{\left(k_{0}\right)}\right)
$$

of uncoded bits, the correct initial state $\mathrm{x}_{0}$ which permits to fulfill the tail-biting boundary condition $\mathbf{x}_{0}=\mathbf{x}_{N}$, where $\mathbf{x}_{N}$ is the final state, can be calculated using the state-space representation

$$
\mathbf{x}_{t+1}=\mathbf{A} \mathbf{x}_{t}+\mathbf{B} \mathbf{u}_{t}^{T}
$$

where $\mathrm{x}_{t+1}$ is the state of the encoder at time $t+1, \mathrm{x}_{t}$ is the state at time $t, m$ is the number of memory elements of the encoder, $\mathbf{A}$ is the $(m \times m)$ state matrix and $\mathbf{B}$ denotes the $(m$ $\mathrm{x} k_{0}$ ) control matrix.

The complete solution of (3) is given by the superposition of the zero-input solution $\mathrm{x}_{t}^{[z i]}$ and the zero-state solution $\mathrm{x}_{t}^{[z s]}$. The zero-input solution $\mathrm{x}_{t}^{[z i]}$ is the state achieved after $t$ cycles if the encoding started in a given state $\mathrm{x}_{0}$ and all input bits are zero, whereas the zero-state solution $\mathrm{x}_{t}^{[z s]}$ is the resulting state at time $t$ if the encoding started in the all-zero state $\mathbf{x}_{0}=\mathbf{0}$ and the information word $\mathbf{u}=\left(\mathbf{u}_{0}, \mathbf{u}_{1}, \ldots, \mathbf{u}_{t-1}\right)$ has been the input. If we demand that the state at time $t=N$, that is after $N$ cycles, is equal to the initial state $\mathbf{x}_{0}$, we obtain, from (3), the equation

$$
\left(\mathbf{A}^{N}+\mathbf{I}_{m}\right) \mathbf{x}_{0}=\mathbf{x}_{N}^{[z s]}
$$

where $\mathbf{I}_{m}$ denotes the ( $\left.m \times \mathrm{x} m\right)$ identity matrix.

Provided the matrix $\left(\mathbf{A}^{N}+\mathbf{I}_{m}\right)$ is invertible, the correct initial state $\mathbf{x}_{0}$ can be calculated from the zero-state response $\mathbf{x}_{N}^{[z s]}$. Calculated $\mathrm{x}_{0}$, a valid codeword $\mathrm{v}$ can be obtain using the equation

$$
\mathbf{v}_{t}^{T}=\mathbf{C x}_{t}+\mathbf{D} \mathbf{u}_{t}^{T}
$$

where $\mathbf{u}$ is the input, $\mathbf{C}$ is the $\left(n_{0} \mathbf{x} m\right)$ observation matrix, and $\mathbf{D}$ denotes the $\left(n_{0} \times k_{0}\right)$ transition matrix. After the Turbo Encoder, since the natural coding rate of the turbo encoder is equal to $1 / 3$ or $1 / 2$, depending on the number of data streams in input, a regular puncturing is performed at the output of the constituent encoders in order to obtain higher code-rate values. Afterwards, the encoded sequence is multiplexed and mapped on the constellations. The 4 modulations which are taken into account in this paper, are the classical QPSK and 8PSK, the 16APSK (amplitude and phase shift keying) and the 32APSK, as provided for the DVB-S2 standard. The latter two modulations allow to minimize the effects of the nonlinear distortion due to non-linear transponders.

\section{THE PROPOSED RECEIVERS}

As for the receiver, two specific structures are defined, concerning the two cases described above (see Fig. 3, Fig. 4). Both structures rely on the iterative turbo decoding, which is based on the MAP algorithm. For every structure the canonical hard demapping, the soft demapping and the iterative soft demapping have been realized so as to highlight the improvements due to the use of the different techniques. In the case of soft demapping, the receiver produces a soft information on reliability of both the systematic and the parity bits. The task which is performed by the soft demapper, can be basically described as the provision of a soft information related to the coded bits starting from the received symbol.

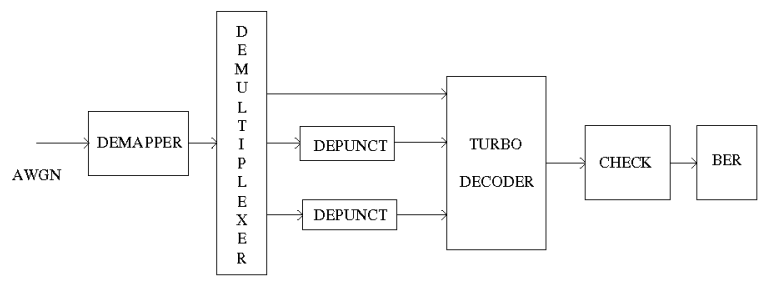

Fig. 3. Receiver with soft demapping and iterative decoding for the proposed system: binary structure

To this end, the complex channel symbols are demapped by a log-likelihood ratio calculation for each of the $M$ coded bits per symbol. Generally, the concept of Log Likelihood 


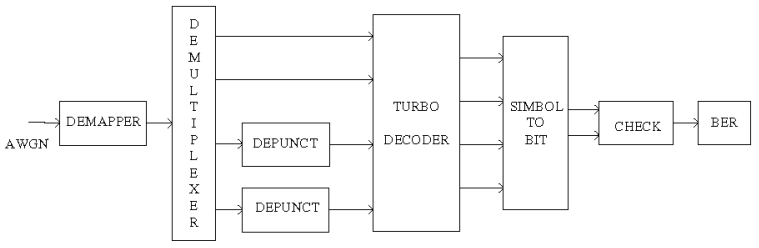

Fig. 4. Receiver with soft demapping and iterative decoding for the proposed system: duobinary structure

Ratios (LLRs) is useful to simplify the passing of information from one component decoder to the other in the iterative turbo decoding [11]. The LLR of a data bit $c_{i}$ is denoted as $L\left(c_{i}\right)$ and is defined as the logarithm of the ratio of the probabilities of the bit taking its two possible values, ie:

$$
L\left(c_{i}\right) \triangleq \ln \frac{P\left(c_{i}=1\right)}{P\left(c_{i}=0\right)}
$$

The sign of the LLR $L\left(c_{i}\right)$ of a bit $c_{i}$ indicates whether the bit is more likely to be 1 or 0 , while the magnitude of the LLR gives an indication of how likely it is that the sign of the LLR gives the correct value of $c_{i}$. This concept proves useful also for the soft demapping. In this case, the demapper produces the conditional LLRs, $L\left(c_{i} \mid \mathbf{z}\right), i=1, \ldots, M$, which can be calculated using the following expression [3]:

$$
L\left(c_{i} \mid \mathbf{z}\right)=\ln \left(\frac{P\left(c_{i}=1 \mid \mathbf{z}\right)}{P\left(c_{i}=0 \mid \mathbf{z}\right)}\right)=L_{a p}\left(c_{i}\right)+
$$

$\ln \left(\frac{\frac{1}{\sigma^{2}} \sum_{j=\operatorname{bin} 1(i)} \exp \left(\left\|\mathbf{z}-y_{j}\right\|^{2}+\sum_{k=\text { other }(j)} L_{a p}\left(c_{k}\right)\right)}{\frac{1}{\sigma^{2}} \sum_{j=\operatorname{bin} 0(i)} \exp \left(\left\|\mathbf{z}-y_{j}\right\|^{2}+\sum_{t=\operatorname{other}(j)} L_{a p}\left(c_{t}\right)\right)}\right)$

where:

- $\sum_{j=b i n 1(i)}$ is referred to the symbols wherein the $i$ th bit is equal to 1 .

- $\sum_{j=b i n 0(i)}$ is referred to the symbols wherein the $i$ th bit is equal to 0 .

- $\mathbf{z}$ is the matched filter output.

- $y_{j}$ is the $j$ th considered symbol.

- $L_{a p}\left(c_{i}\right)$ is the a priori log-likelihood ratio of the bit $c_{i}$.

- $\sum_{k=\text { other }(j)}$ is referred to the other bits of the considered symbol that are equal to 1 .

- $\sum_{t=o t h e r(j)}$ is referred to the other bits of the considered symbol that are equal to 0 .

These values are called the a posteriori log-likelihood ratios. After the demapper, these soft values are passed to the iterative turbo decoder composed by two component decoders that are connected by the interleavers. At each new iteration, the iterative structure allows to provide an additional information to the first decoder in order to obtain a more accurate set of soft outputs, which are then used by the second decoder as a priori information. The binary structure works in the logarithmic domain while the duo-binary one works in the domain of the probabilities. In the latter case, since the structure works at "symbol level", the MAP algorithm is modified for producing the a posteriori probability of each systematic symbol and, moreover, also in the decoder, the permutation function of the interleavers and of the deinterleavers is performed on the two levels. In case of iterative soft demapping, the decoding algorithm is properly modified to produce also the extrinsic information on the parity bits. The extrinsic information is obtained subtracting from the a posteriori information, the $a$ priori information and the received systematic channel input. This relation is valid for systematic and non systematic coded bits. After a fixed number of turbo decoder iterations, through the deinterleaver, the extrinsic information of coded bits at the output of the turbo decoder are fed back to the input of the soft demapper as the a priori information, $L\left(c_{i}\right)$, for the next receiver iteration. The demapper can utilize the a priori information received from the decoder to calculate improved a posteriori values, $L\left(c_{i} \mid \mathbf{z}\right)$, which are passed as extrinsic values to the decoder for further iterative decoding steps [3].

\section{SimUlation RESUlTS}

In order to demonstrate the effectiveness of the proposed receiver, a set of computer simulations was performed.

For all simulations, the used decoding algorithm in the Turbo Decoder has been the Log-MAP algorithm since it permits to achieve very good performance but with a complexity lower than the MAP algorithm while the considered channel has been an AWGN one.

As shown before, we considered two structures: the former used for every code rate required by DVB-S2 standard and the latter utilized only when code rates are higher than $1 / 2$. In the following we will separate these two cases also for simulation results. In every graph we will indicate if the simulation results are referred to a binary structure or to a duo-binary structure, the used Frame Length (FL), the considered constellation and the number of iterations of the turbo decoder. In those graphs where a performance comparison is described, also the type of considered demapping (Hard, Soft or SoftIterative) is indicated. Since the Turbo encoder structure was based on the DVB-RCS standard, the considered Frame Lengths are all multiple of 8 as provided for such system. In Fig. 5 we report

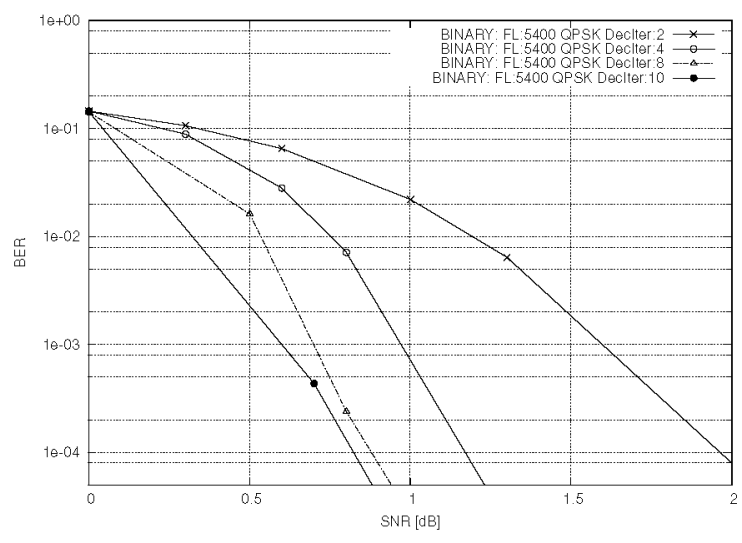

Fig. 5. Performance in terms of Bit Error Rate (BER) for QPSK, rate 1/3, in case of soft demapping and iterative decoding 


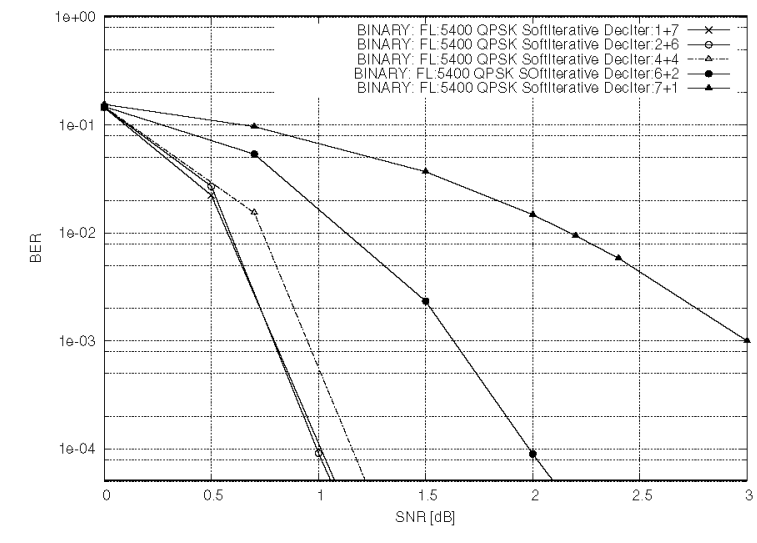

Fig. 6. Performance in terms of Bit Error Rate (BER) for QPSK, rate 1/3, in case of iterative soft demapping and iterative decoding: $1+7,2+6,4+4,6+2$ $7+1$ iterations

the performance in terms of Bit Error Rate (BER) in case of QPSK modulation, code rate equal to $1 / 3$, soft demapping and iterative decoding. The performance has been analyzed for different values of the number of iterations of the turbo decoder. As the signal to noise ratio (SNR) increases, the value of BER decreases and the gain is more evident as the number of the iterations passes from 2 to 4,8 and 10 iterations. We can observe that no further sensible benefit is achieved for values which are higher than 8: this happens because, beyond this value, the additional information which can be obtain from the extrinsic information is lower. In Fig. 6 we show the performance in terms of Bit Error Rate (BER) in case of QPSK modulation, code rate equal to $1 / 3$, iterative soft demapping and iterative decoding. In this case, we report the results as the total number of iterations changes. As "total number" we mean the sum of the number of iterations of the turbo decoder before the outer demapping iteration and of the ones after the external iteration. We can observe which is the best strategy for realizing the iterative demapping. Indeed, the best results are obtained when few turbo iterations are performed before the external iteration of demapping and many turbo iterations are carried out after the iteration of demapping. In Fig. 7 the performance comparison between hard demapping, soft demapping and iterative soft demapping in terms of Frame Error Rate (FER) for QPSK modulation, code rate equal to $1 / 3$, is reported. The considered total number of iterations is equal to 8 because, as proven above, this value turns out to be the most suitable choice as for a turbo decoding. As Frame Error Rate (FER) we mean the wrong frames rate compared with the total number of frames, where a "wrong frame" is that where at least one bit is detected erroneously. In this case no advantage is due to the iterative demapping in comparison with the non iterative approach: this result depends on the mapping strategy which has been adopted and on the number of demapping iterations. The Gray mapping determines the independence of the bits in phase and in quadrature and so it is not possible to take advantage of the joint information of the bits belonging to the same symbol. A better performance

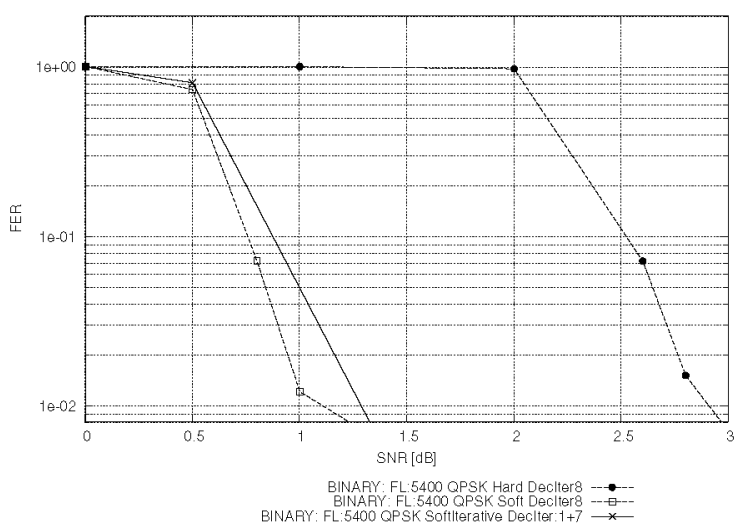

Fig. 7. Performance comparison in terms of Frame Error Rate (FER) for QPSK, rate $1 / 3$, between hard demapping, soft demapping and iterative soft demapping

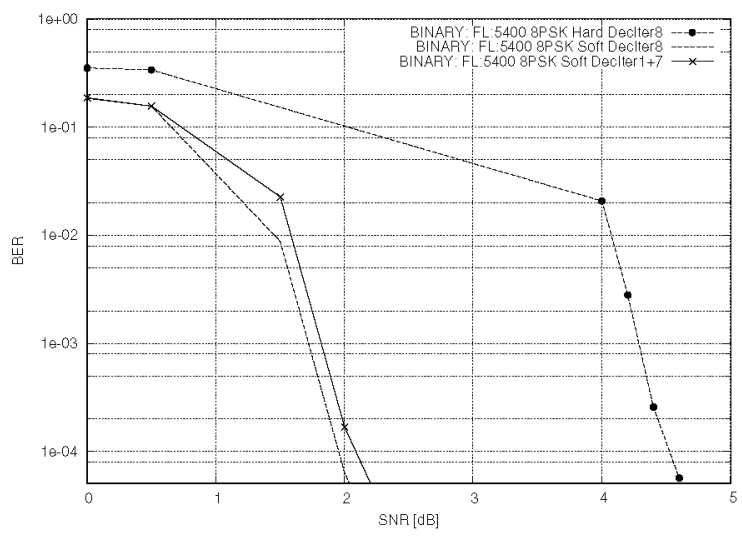

Fig. 8. Performance comparison in terms of Bit Error Rate (BER) for 8PSK, rate $1 / 3$, between hard demapping, soft demapping and iterative soft demapping

could be obtained if a non Gray approach was considered. We report also the performance comparison between hard demapping, soft demapping and iterative soft demapping in terms of Bit Error Rate (BER) in case of 8PSK modulation, code rate equal to $1 / 3$ : as it can be seen in Fig. 8 , the results are similar to the QPSK case, for what concerns the demapping strategies. Finally, we show in Fig. 9 and in Fig. 10 the performance comparison in terms of Bit Error Rate (BER) in case of 16APSK constellation, code rate $2 / 3$ and of 32 APSK constellation, code rate $4 / 5$. The soft demapping produces some advantages compared to hard approach, as described in the previous results. As for the iterative soft demapping, instead, also in case of higher modulation, we do not obtain a performance improvement. Therefore, we deem that the iterative demapping strategies need to further elaborations to reach a better and clearer conclusion.

In the following, we will analyze some simulation results for the duo-binary structure, as well. As shown before, this structure uses the duo-binary turbo codes: hence it was necessary to modify both the decoding algorithm and the soft demapper. In Fig. 11 and in Fig. 12, we show the comparison in terms of 


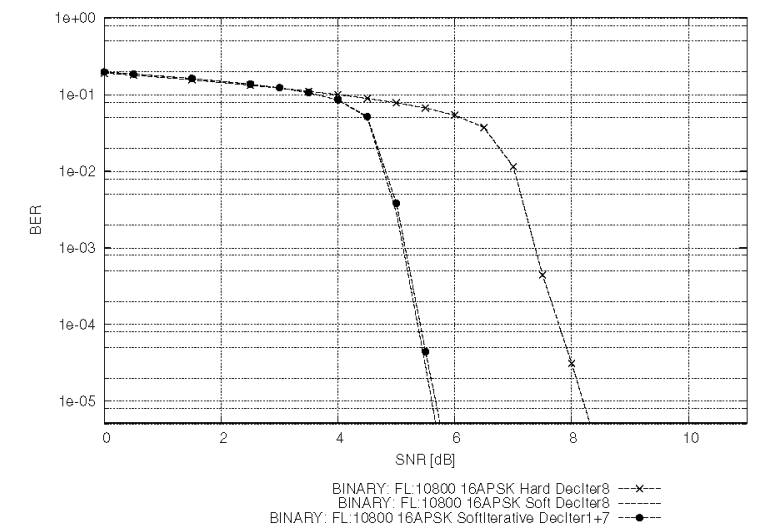

Fig. 9. Performance comparison in terms of Bit Error Rate (BER) for 16APSK, rate $2 / 3$, between hard demapping, soft demapping and iterative soft demapping

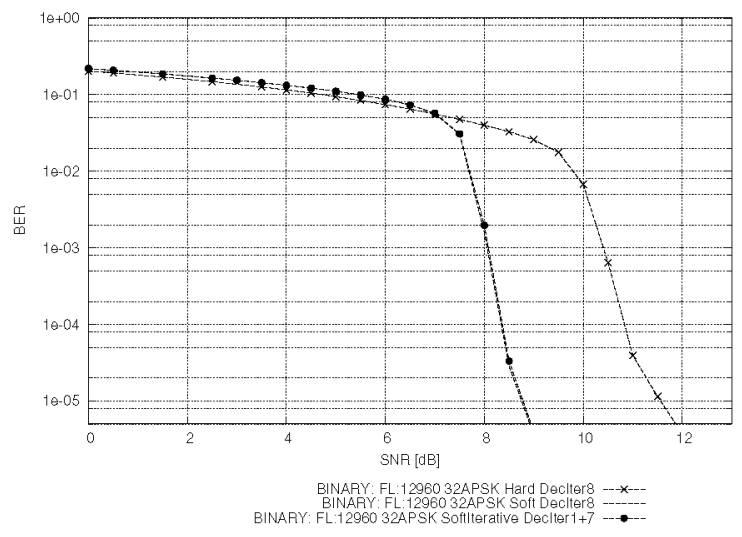

Fig. 10. Performance comparison in terms of Bit Error Rate (BER) for $32 \mathrm{APSK}$, rate $4 / 5$, between hard demapping, soft demapping and iterative soft demapping

Bit Error Rate (BER) and of Net Throughput between hard demapping and soft demapping for QPSK modulation and code rate equal to $2 / 3$. As Net Throughput we mean the data rate obtained by the corrected transmissions for every frame. Also in this case, the results confirm what already shown for the binary structure: the soft demapping behaves better than the hard one, i.e., also in the duo-binary case the soft demapping is beneficial.

\section{CONCluding Remarks}

In this paper we have proposed a novel detection strategy for satellite communications. The advanced detectors which have been described, are based on an iterative turbo decoding and on iterative and non iterative soft demapping approach. The adoption of the soft demapping has presented a remarkable performance gain in comparison with the canonical hard strategy in all cases which have been analyzed.

\section{REFERENCES}

[1] DVB-S2 European Standard, Digital Video Broadcasting (DVB); Second generation framing structure, channel coding and modulation system for Broadcasting, Interactive Services, News Gathering and other broadband satellite applications, ETSI EN 302 307, v. 1.1.2, 2006-06.

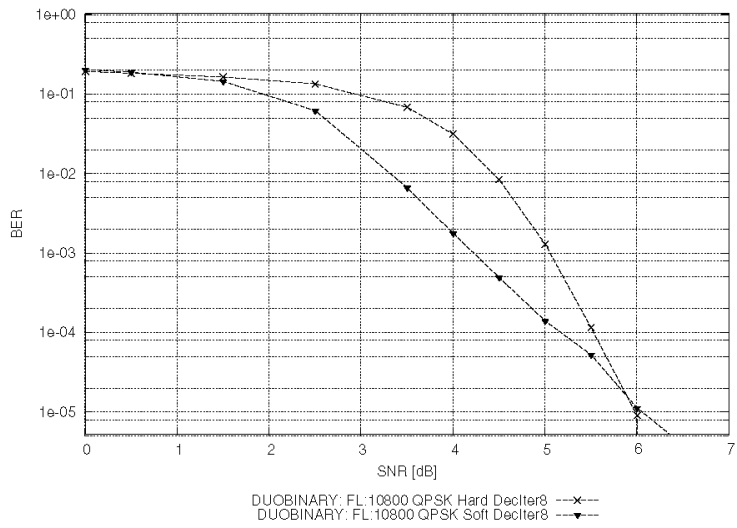

Fig. 11. Performance comparison in terms of Bit Etror Rate (BER) for QPSK, rate 2/3, between hard demapping and soft demapping

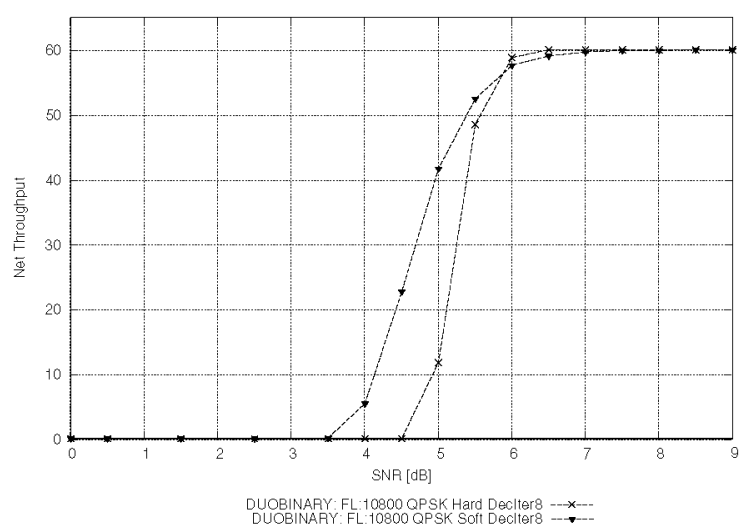

Fig. 12. Performance comparison in terms of Net Throughput for QPSK, rate $2 / 3$, between hard demapping and soft demapping

[2] DVB-S2 Technical Report, Digital Video Broadcasting (DVB) User guidelines for the second generation system for Broadcasting, Interactive Services, News Gathering and other broadband satellite applications (DVB-S2), ETSI TR 102376 , v. 1.1.1, 2005-02.

[3] S. ten Brink, J. Speidiel and Yan Ran-Hong "Iterative Demapping and Decoding for multilevel modulation" IEEE Global Telecommunications Conference, Vol. 1, pp. 579-584, 1998.

[4] DVB-RCS European Standard, Digital Video Broadcasting ( DVB); Interaction channel for satellite distribution system, ETSI EN 301790 ,v. 1.4.1, 2005-09.

[5] C. Berrou, A. Glavieux and P. Thitimajshima "Near Shannon limit errorcorrecting coding and decoding: Turbo codes" Proc. 1993 Int. Conf. on Communications (ICC'93), Washington DC.

[6] C. Berrou and C. Douillard "Turbo Codes With Rate- $\mathrm{m} /(\mathrm{m}+1)$ Constituent Convolutional Codes" IEEE Transactions on Communications, Vol. 53, No.10, pp. 1630-1638, October 2005.

[7] C. Berrou, M. Jézéquel, C. Douillard and S. Keroudan "The advantages of non-binary turbo codes" Proc.Information Theory Workshop, Cairns, Australia, pp. 61-63, September, 2001

[8] C. Berrou and M. Jézéquel "Non binary convolutional codes for turbo coding" Electronics Letters, Vol. 35, pp. 39-40, January, 1999

[9] C. Weiss, C. Bettstetter, S. Riedel and D.J. Costello "Turbo Decoding with Tail-Biting Trellises" IEEE, 1998.

[10] C. Weiss, C. Bettstetter "Code Construction and Decoding of Parallel Concatenated Tail-Biting Codes" IEEE Transactions on Information Theory, 2001.

[11] J. P. Woodard and L. Hanzo "Comparative study of Turbo Decoding Techniques: an Overview", ed. IEEE,2000. 